\title{
Fault Ride Through Strategy for Offshore Wind Farm Integration System via MMC-HVDC
}

\author{
Zhang Lijun ${ }^{1}$, Zhong Yujun ${ }^{2}$, Chen Rui ${ }^{3}$, Sun Yikai ${ }^{1}$, Zhang Jing ${ }^{4}$ and $\mathrm{Jia} \mathrm{Ke}^{3}$ \\ ${ }^{1}$ State Grid Zhejiang Economy Research Institute, Hangzhou, Zhejiang, China \\ ${ }^{2}$ State Grid Zhoushan Power Supply Company, Zhoushan, Zhejiang, China \\ ${ }^{3}$ School of Electrical \&Electronic Engineering, North China Electric Power University, Beinong Road, Beijing,China \\ ${ }^{4}$ State Grid Zhejiang Electric Power Co., Ltd., Hangzhou, Zhejiang, China
}

\begin{abstract}
When offshore wind power is transmitted to ac grid through MMC-HVDC, the current and voltage will be quite different from those in traditional ac grid during grid side fault. This paper sets up an offshore wind farm integration system via MMC-HVDC and designs control strategies for each unit in the system. The fault ride through strategy of the system is proposed and its effectiveness has been verified. Thus, the AC bus voltage on wind farm side will stay stable during AC side fault. Once the chopper resistance is set properly, the output power and current of the wind farm can basically remain unchanged, which can successfully achieve fault isolation. The simulation results based on PSCAD have verified the theoretical analysis.
\end{abstract}

\section{INTRODUCTION}

With the rapid development of renewable power generation, offshore wind power installed capacity has reached $14384 \mathrm{MW}$ in 2016 [1]. Because of its flexibility and lower cable power losses, MMC-HVDC is widely used to transmit electricity generated by offshore wind farm [2]. However, when fault occurs on AC grid side of MMC-HVDC, excess power will accumulate on DC transmission line, causing DC voltage increases rapidly. Besides, the negative-sequence current during asymmetrical fault will cause the DC voltage and active power fluctuate. Thus, the FRT strategy should be proposed to ensure the stable operation of wind farm integration system during AC side fault.

Nowadays, the FRT strategy of MMC-HVDC connected wind farm integration system consists of two methods: The first one is to reduce the output power of wind farm during AC side fault by controlling the voltage or frequency of the wind farm side converter [3-6]. This method does not require additional equipment, but overrange frequency or voltage changes may have a great impact on wind turbine. Thus, the voltage or frequency can only be limited within a certain range [7]. The second method is to achieve FRT of the system by using the chopper resistance, consuming the excess power on DC transmission system as heat during a fault period [8-9]. This method is simple and feasible. And it can solve the problem of active power difference between the AC grid and the wind farm of the system during $\mathrm{AC}$ side fault
[10]. From the discussions above, the existing studies haven't probed the application of fault isolation of the chopper resistance considering different control strategies work together, which will lay a foundation for further application of the resistance.

This paper proposes a fault ride through strategy for offshore wind farm integration system. The main contributions of this paper are: 1) Combined with the structure of the system, this paper sets up models for MMC-HVDC and wind farms in offshore wind farm integration system. 2) Considering the FRT requirement of renewable energy grid-connected system, this paper proposes a FRT control strategy by using the chopper resistance for MMC-HVDC connected wind farm integration system. The AC fault isolation function of the chopper resistance is deduced in this paper, which will provide theoretical support for the application of the FRT strategy in the future.

\section{SYSTEM STRUCTURE}

A MMC-HVDC connected wind farm system is built in this paper. Figure 1 shows the single-line structure of the system.

\footnotetext{
*Corresponding author: zhanglijun@zj.sgcc.com.cn, zyujun_zs@163.com, 303298178@qq.com, sun_yikai@zj.sgcc.com.cn,
} eejzhang@126.com,ke.jia@ncepu.edu.cn 


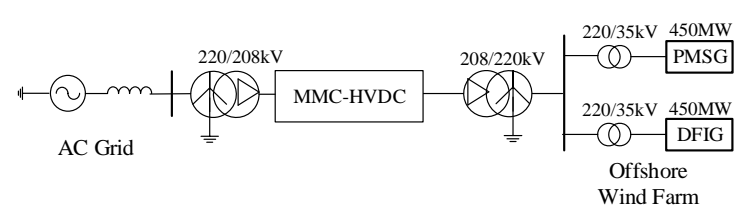

Figure 1: Single-line structure of the offshore wind farm integration system via MMC-HVDC transmission.

The electric power generated by the wind farm is collected by the WFMMC. Then, it is transmitted to the main AC grid through a 200km cable and the GSMMC.

\section{SYSTEM CONTROL}

The control strategy is essential for an offshore wind farm integration system to operate stably. For a stable operation, the power generated by the wind farm must be stable and the DC voltage of the transmission line must be maintained at a constant value, while the frequency and $\mathrm{AC}$ voltage of the wind farm must be stable to ensure the power generated by the wind farm stayed constant. Thus, it is important to study the control of each part of the whole system during fault and stable operation.

\subsection{MODEL AND CONTROL OF PMSG}

The typical structure of PMSG (Permanent Magnet Synchronous Generator) is showed in Figure 2.

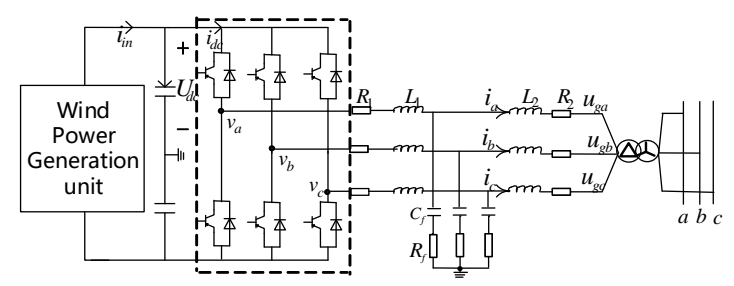

Figure 2: Typical structure of PMSG.

In order to suppress the fault current fluctuation better when unbalanced fault occurs on the grid, the grid side converter adopts negative-current-suppressed strategy. The reference value for the converter control system is defined as

$$
\left\{\begin{array}{l}
i_{\mathrm{d}}^{+*}=2\left(e_{\mathrm{d}}^{+} P^{*}+e_{\mathrm{q}}^{+} Q^{*}\right) /\left[3\left(e_{\mathrm{d}}^{+}\right)^{2}+3\left(e_{\mathrm{q}}^{+}\right)^{2}\right] \\
i_{\mathrm{q}}^{+*}=2\left(e_{\mathrm{q}}^{+} P^{*}-e_{\mathrm{d}}^{+} Q^{*}\right) /\left[3\left(e_{\mathrm{d}}^{+}\right)^{2}+3\left(e_{\mathrm{q}}^{+}\right)^{2}\right] \\
i_{\mathrm{d}}^{-*}=i_{\mathrm{q}}^{-*}=0
\end{array}\right.
$$

In the above equation, $e_{\mathrm{d}}^{+}, e_{\mathrm{q}}^{+}$represent for the threephase voltage at the exit of PMSG. $P^{*}, Q^{*}$ are the active and reactive power reference values of the control system during fault crossing process.

\subsection{MODEL AND CONTROL OF DFIG}

The typical structure of DFIG (Double-Fed Induction Generator) is showed in Figure 3.

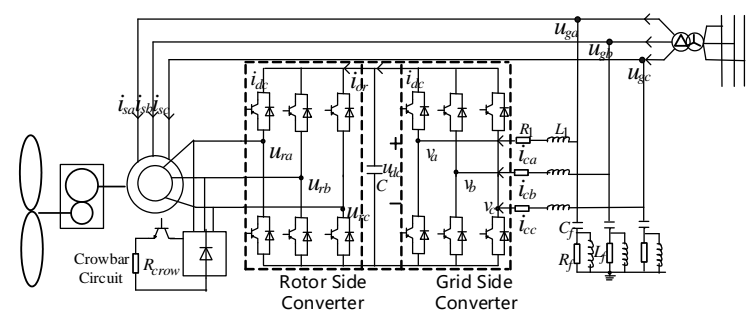

Figure 3: Typical structure of DFIGURE

During a fault, the voltage falls down, which will generate great voltage and current in the rotor windings, and it will damage the rotor side converter. In this paper, the crowbar circuit can make sure that the wind turbine has the ability to realize the fault crossing process, coordinating with the negative current-suppressed strategy of the grid side converter.

\subsection{MODEL AND CONTROL OF HVDC}

The structure of MMC-HVDC is shown as follows.

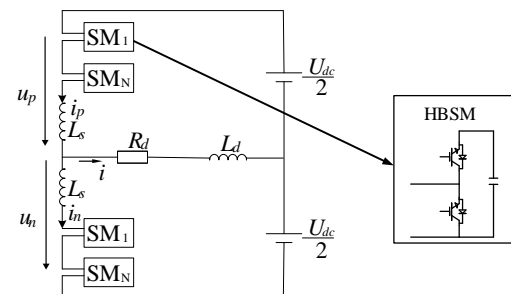

Figure 4: Typical structure of MMC-HVDC.

The MMC converter consists of two arms, which contains $\mathrm{N}$ half bridge submodules in series connection respectively.

\subsubsection{CONTROL STRATEGY OF GSMMC}

For a stable operation, the DC voltage and output reactive power of GSMMC must remain constant. So the grid side MMC applies double closed loop vector control strategy based on the oriented grid voltage to make GSMMC a power balance point.

When $\mathrm{d}$-axis is orientated at the grid voltage, the current inner loop uses PI controller to achieve a complete decoupling of $\mathrm{d}, \mathrm{q}$ axis for the currents, which can be expressed as:

$$
\left\{\begin{array}{l}
u_{d}^{*}=-\left(L \frac{d i_{d}}{d t}+R i_{d}\right)=\left(K_{p}+\frac{K_{i}}{s}\right)\left(i_{d}^{*}-i_{d}\right) \\
u_{q}^{*}=-\left(L \frac{d i_{q}}{d t}+R i_{q}\right)=\left(K_{p}+\frac{K_{i}}{s}\right)\left(i_{q}^{*}-i_{q}\right)
\end{array}\right.
$$

For the voltage outer loop, this paper uses DC voltage controller to control system's active power. When the offshore wind farm integration system via MMC-HVDC operates stably, GSMMC must fix the DC voltage as a power balance point in order to ensure the stable operation of the system. The structure of the controller is shown as below. 


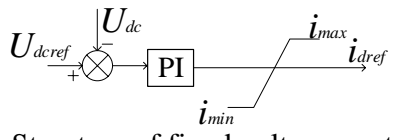

Figure 5: Structure of fixed voltage controller.

Thus, the overall control strategy of GSMMC is shown in Figure 6.

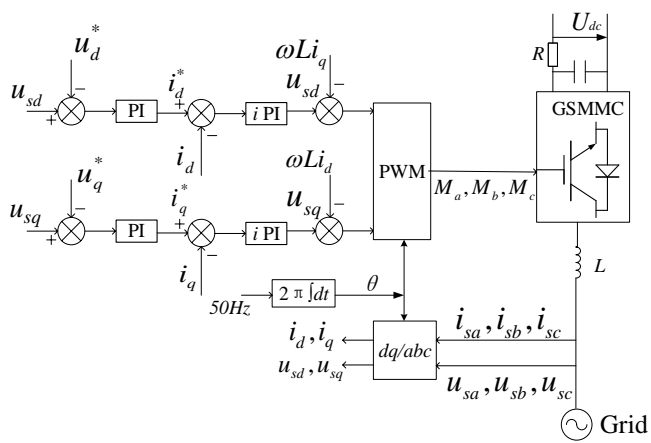

Figure 6: Control strategy of GSMMC.

\subsubsection{CONTROL STRATEGY OF WFMMC}

WFMMC has a same transient mathematical model as GSMMC. Here, we don't discuss it any more.

As the wind farm cannot hold a stable frequency and voltage during fault, WFMMC applies Vf control to fix the wind farm's voltage and frequency. In a Vf control strategy, the voltage reference value can be calculated by the following expressions:

$$
\left\{\begin{array}{l}
u_{\text {dref }}=k_{p}\left(U_{\text {acref }}-U_{s}\right)+k_{i} \int\left(U_{\text {acref }}-U_{s}\right) d t+0.5 U_{d c} \\
u_{\text {qref }}=0 \\
\theta_{\text {passive }}=2 \pi f_{0}+\theta_{0}
\end{array}\right.
$$

Where $u_{\text {dref }}, u_{\text {qref }}$ are the reference voltages. $\theta_{\text {passive }}$ is the reference phase, while $\theta_{0}$ is the initial phase. $f_{0}$ is the fixed frequency of $\mathrm{AC}$ voltage. $U_{s}$ is the wind farm side AC bus voltage, while $U_{\text {acref }}$ is defined as its reference value. $U_{d c}$ is the output DC voltage of WFMMC.

According to (4), the control strategy is shown as following.

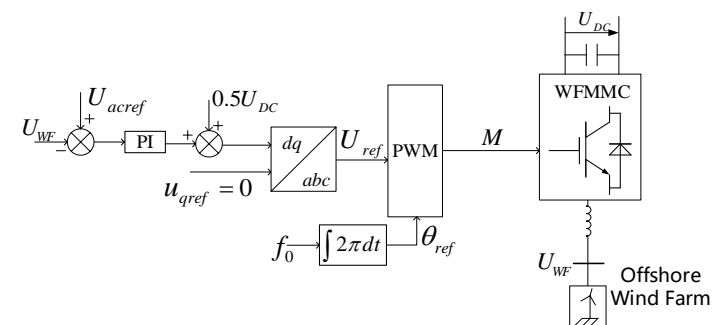

Figure 7: Control strategy of WFMMC.

\subsubsection{FRT STRATEGY OF MMC-HVDC}

When fault occurs on grid side of MMC-HVDC, the power output capacity of GSMMC will decrease, while the power transmission ability of WFMMC will not be affected. During severe fault, the active power at both ends cannot be balanced. DC voltage will be too high to burn the DC transmission line. Thus, it is necessary to take measures to limit the rapid increase of the DC voltage.

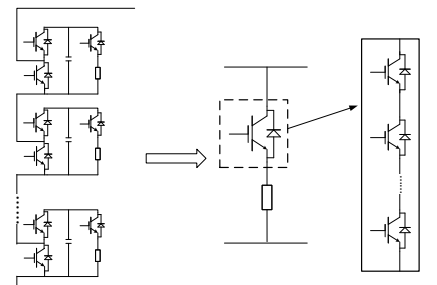

Figure 8: Topology of chopper resistance.

When the output capacity of GSMMC decreases to 0 during severe fault, the chopper resistances are equivalent to a large discharge resistance between the parallel DC transmission lines. When fault occurs, the chopper resistance will operate and it can consume the extra power accumulated on the transmission line. Moreover, if the capacity of resistance is selected properly, MMC-HVDC transmission system can fully achieve fault isolation, ensuring the stable operation of the wind farm and WFMMC. The topology of the chopper resistance is shown in Figure 8.

When the grid side fault brings decrease to the system voltage, the ability for GSMMC to output active power will be weakened. Ignore losses of the converter and the transmission line, we can obtain the expression of the chopper resistance.

$$
R_{\text {crossing }}=\frac{\left(V_{N, \text { total }}^{*}\right)^{2}}{P_{W F}-P_{\text {Grid }}^{f}}
$$

$P_{W F}$ is the rated power of the wind farm, $P_{\text {Grid }}{ }^{f}$ is the output power from GSMMC during AC fault. $V_{N \text {,total }}^{*}=k V_{N}{ }^{*}, k$ is the operating threshold of the chopper resistance, which is set at $1.1 . \mathrm{V}_{\mathrm{N}}{ }^{*}$ is the rated voltage of the DC line.

To ensure the system achieve FRT when the voltage drops on grid side, the most severe fault type is considered in this paper. Thus, the rated power calculation formula of the chopper resistance is defined as:

$$
P_{\text {crossing }}=\frac{\left(V_{N, \text { total }}^{*}\right)^{2}}{R_{\text {crossing }}}
$$

\section{FAULT ISOLATION OF RCROSSING}

The power flow of the wind farm integration system during fault is shown in Figure 9.

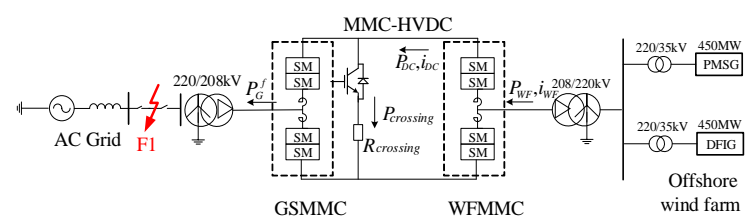

Figure 9: Simulation system of offshore wind farm integration system via MMC-HVDC transmission. 
When the system voltage falls down because of AC grid side fault, the chopper resistance will be put into operation. The power consumption of the resistance while the wind farm output power is rated power can be expressed as:

$$
P_{\text {crossing }}=P_{w}-P_{G}^{f}=\frac{\left(V_{N, \text { total }}^{*}\right)^{2}}{R_{\text {crossing }}}
$$

Ignore the loss of the two converters during fault, $P_{w}=P_{D C}=P_{G}$.

When fault occurs on grid side, the power accumulated on DC transmission lines can be calculated by

$$
P_{D C}=P_{\text {crossing }}+P_{G}^{f}=P_{w}
$$

From the above equation, $P_{D C}$ will basically remain the same during fault due to the input of the chopper resistance.

$$
P_{D C}=\frac{U_{D C}^{2}}{R_{D C}}
$$

From the above equation, we can draw a conclusion that DC voltage will remain as 1.1p.u. during AC side fault. Thus, the current of the DC transmission line will also stay at a constant value.

From the model of MMC,

$$
C \frac{d U_{D C}}{d t}=\left[\begin{array}{c}
\lambda M \cos (\omega t+\delta) \\
\lambda M \cos \left(\omega t+\delta-120^{\circ}\right) \\
\lambda M \cos \left(\omega t+\delta+120^{\circ}\right)
\end{array}\right]\left[\begin{array}{c}
i_{W F a} \\
i_{W F b} \\
i_{W F c}
\end{array}\right]-i_{d c, \text { line }}
$$

It can be concluded that the AC current from GSMMC will be basically unchanged when the modulation ratio is constant. Thus, MMC-HVDC plays a role of isolation to the AC side fault for the wind farm. As $R_{\text {crossing }}$ is put into the system from time to time, the power on DC transmission line will be affected, thus affecting the DC voltage. However, due to the Vf control strategy, the AC voltage of wind farm will remain unchanged when bus current is limited within the limiting range.

\section{SIMULATION RESULTS}

In order to verify the analysis in section IV, a simulation system is built in PSCAD/EMTDC. During the simulation period, the $\mathrm{AC}$ side fault occurs at $4.2 \mathrm{~s}$ on the middle of AC transmission line. The duration time is set at $0.1 \mathrm{~s}$.

Figure 11 shows the performance of the offshore wind farm integration system when phase A is grounded at the middle of the AC transmission line.
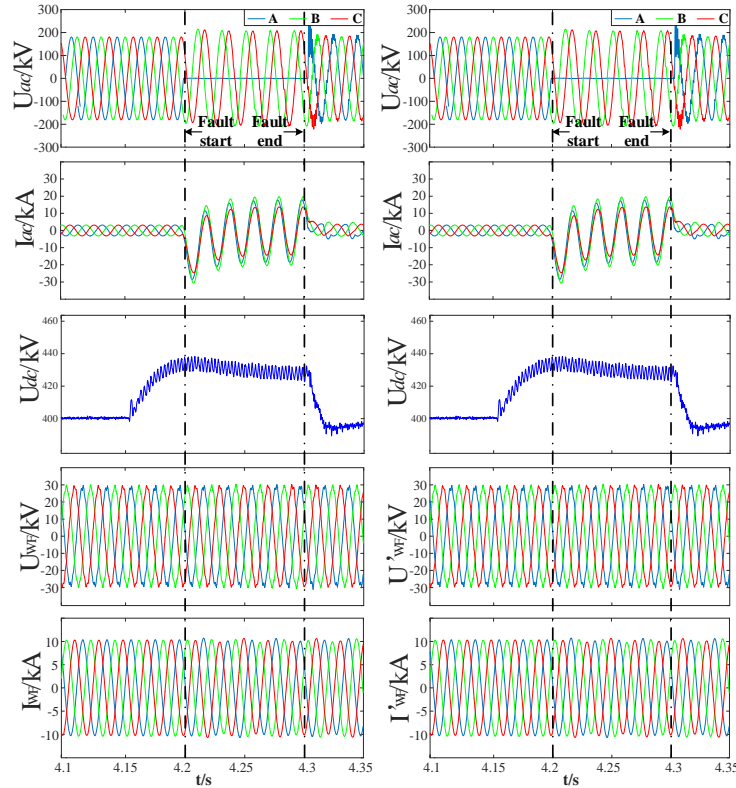

(a)Mixed wind farm (DFIG and PMSG) (b) PMSG wind farm

Figure 10: Performance of offshore wind farm integration system during A-phase-to-ground fault.

Comparing Figure 10(a) with (b), it can be found that when a single-phase-to-ground fault occurs on the AC grid side of MMC-HVDC, the fault currents provided by MMC-HVDC and the wind farm are the same under different types of wind farms. With the operation of the FRT strategy, the DC voltage of MMC-HVDC is limited at its threshold 1.1p.u. Because the wind farm side converter adopts the Vf control strategy so that the voltage of the AC bus of the wind farm remains unchanged, the wind farm can maintain the stable operation of the original state during fault. Finally, it can be obtained that the output AC current of the wind farm remains basically unchanged.

\section{CONCLUSION}

The control strategies of offshore wind farm integration system via MMC-HVDC are discussed in this paper. The FRT strategy of MMC-HVDC is proposed. After setting up the system in PSCAD, the fault characteristics, as well as the performance of the chopper resistance are analysed. Simulations have verified the isolation function of the proposed FRT strategy, which will lay a foundation for future studies of the offshore wind integration system.

\section{ACKNOWLEDGEMENTS}

This work was financially supported by Science and Technology Project of State Grid Zhejiang Electric Power Co., Ltd. (5211JY16000U).

\section{REFERENCES}

1. Global Wind Power Statistics, 2016. GWEC, 2017.

2. $\mathrm{Xu}$, Lie, and Bjarne R. Andersen. "Grid connection of large offshore wind farms using HVDC." Wind Energy 9.4 (2006): 371-382. 
3. W. Li, J. Lv, G. Shi, X. Cai and Y. Chi, "Improved AC fault ride through control strategy for MTDC system with offshore wind farms," 2014 International Conference on Power System Technology, Chengdu, 2014, pp. 2409-2419.

4. Y. Yu, Z. Xu and T. An, "Fault ride-through strategy for fully rated converter wind turbines connected to the grid via MMC-HVDC transmission," 12th IET International Conference on $\mathrm{AC}$ and $\mathrm{DC}$ Power Transmission (ACDC 2016), Beijing, 2016, pp. 1-5.

5. Y. Jing, R. Li, L. Xu and Y. Wang, "Enhanced AC voltage and frequency control on offshore MMC station for wind farm," in The Journal of Engineering, vol. 2017, no. 13, pp. 1264-1268, 2017.

6. L. Xuan, S. Qiang, L. Wenhua and M. Yulong, "Study on fault ride-through capability of wind farm integration using MMC-HVDC," 2014 International Conference on Power System Technology, Chengdu, 2014, pp. 2596-2601.

7. L. Xu and L. Yao, "DC voltage control and power dispatch of a multi-terminal HVDC system for integrating large offshore wind farms," in IET Renewable Power Generation, vol. 5, no. 3, pp. 223233, May 2011.

8. S. Cao, W. Xiang, L. Yao, B. Yang and J. Wen, "AC and DC fault ride through hybrid MMC integrating wind power," in The Journal of Engineering, vol. 2017, no. 13, pp. 828-833, 2017.

9. C. Feltes, H. Wrede, F. W. Koch and I. Erlich, "Enhanced Fault Ride-Through Method for Wind Farms Connected to the Grid Through VSC-Based HVDC Transmission," in IEEE Transactions on Power Systems, vol. 24, no. 3, pp. 1537-1546, Aug. 2009.

10. Y. Li et al., "Study on AC-side dynamic brakingbased fault ride-through control for islanded renewable energy system with grid-connected VSCHVDC transmission," 2017 Chinese Automation Congress (CAC), Jinan, 2017, pp. 6108-6111. 